\title{
Processo analítico hierárquico na identificação de áreas favoráveis ao agroecossistema cafeeiro em escala municipal
}

\author{
Marco Aurélio Barros ${ }^{(1)}$, Maurício Alves Moreira(1) e Bernardo Friedrich Theodor Rudorff(1)
} (1)Instituto Nacional de Pesquisas Espaciais, Av. dos Astronautas, 1.758, Jardim Granja, CEP 12227-010 São José dos Campos, SP. E-mail:
aurelio@dsr.inpe.br, mauricio@dsr.inpe.br, bernardo@dsr.inpe.br

\begin{abstract}
Resumo - O objetivo deste trabalho foi delimitar áreas favoráveis ao agroecossistema cafeeiro, em quatro municípios do Estado de Minas Gerais, pela aplicação do processo analítico hierárquico (AHP). Uma função de ponderação aritmética foi obtida, com base nas premissas de favorabilidade à cafeicultura, considerando-se as seguintes variáveis: solo, declividade, orientação de vertentes, altimetria e as possíveis áreas de preservação permanente. Essa função permitiu combinar as condições adequadas ao cultivo do café e ressaltar as áreas com maior favorabilidade. Foi verificado que os quatro municípios diferem entre si quanto à favorabilidade ao agroecossistema cafeeiro; porém, ao se considerar apenas as áreas cultivadas com café, foi verificado que os municípios de Boa Esperança e Cristais não diferem entre si.
\end{abstract}

Termos para indexação: meio ambiente, ecossistema, cultura do café.

\section{Analytical hierarchical process to identify favorable areas to the coffee crop agroecosystem at municipal scale}

\begin{abstract}
The objective of this work was to delimitate favorable areas to the coffee agroecosystem, in four municipalities of Minas Gerais State, Brazil, using the analytical hierarchical process (AHP). An arithmetic pondering function was obtained, based on the favorability premises to coffee crop production, with regard to the following variables: soil, slope, slope aspect, altimetry, and areas that should be under permanent protection. This function allowed to combine the adequate conditions to coffee crop cultivation and gave emphasis to the most favorable areas. It was observed that all four municipalities were different in terms of their favorability to the coffee agroecosystem; however, when considering only the coffee cultivated areas, it was observed that the municipalities of Boa Esperança and Cristais were not significantly different.
\end{abstract}

Index terms: environment, ecosystem, coffee crop.

\section{Introdução}

O aumento da produção agrícola mundial vem provocando uma crescente preocupação com a salubridade alimentar e a conservação dos recursos naturais do planeta. Nesse sentido, tem-se buscado métodos para modelar o meio físico e, com isso, predizer situações adversas nas quais o meio ambiente pode estar exposto. Nesse processo, a ciência da geoinformação vem contribuir na convergência de diversas áreas do conhecimento, como geografia, agronomia e informática, para o diagnóstico e solução dos problemas enfrentados pelo homem, como também na simulação de cenários, a partir de determinadas variáveis ambientais (Câmara et al., 2007).
Trabalhos científicos demonstram a importância da utilização de geotecnologias no estudo da aptidão agrícola de terras, manejo de bacias hidrográficas, estudo e modelagem de relevo, análise ambiental de espaços geográficos e zoneamento ecológico econômico. Esses trabalhos enfatizam a importância da utilização de dados orbitais, como suporte à extração de informações temáticas (Felgueiras \& Erthal, 1988; Kurkdjian et al., 1992; Venturieri, 1995; Crepani et al., 2001; Crepani \& Medeiros, 2004).

O processo analítico hierárquico (AHP) tem sido aplicado em trabalhos relacionados à agricultura e gestão de recursos naturais (Alphonce, 1997; Guo \& He, 1999; 
Thirumalaivasan et al., 2003; Zhang et al., 2004; Li et al., 2005; Karami, 2006). Na agricultura, destacam-se estudos para determinar as áreas aptas a cultivos, melhores métodos de irrigação e áreas favoráveis à conservação e ao desenvolvimento de atividades agropecuárias.

O processo analítico hierárquico, desenvolvido por Saaty (1986), é uma técnica de tomada de decisão com múltiplos critérios, em que um problema complexo possui uma hierarquia de solução a partir das variáveis utilizadas nessa solução (Alphonce, 1997). Entre os trabalhos desenvolvidos com o AHP, cita-se a seleção do melhor equipamento de irrigação em fazendas no Irã, país com grande escassez de recursos hídricos (Karami, 2006).

Li et al. (2005) estudaram o agroecossistema de uma província chinesa, na determinação de áreas próprias para o cultivo do arroz, visando a maximização do uso dos recursos naturais de forma menos agressiva ao meio ambiente, para subsidiar a solução de problemas sociais, econômicos e ecológicos.

Seguindo a tendência atual de estudos dos ecossistemas agrícolas que auxiliam na delimitação de áreas favoráveis à exploração agropecuária, realizouse a aplicação do método AHP para determinar regiões favoráveis à cafeicultura, tendo-se respeitado o conceito de agroecossistema. Com base nesse conceito, priorizase a exploração dos recursos naturais de forma mais sustentável, respeitam-se suas limitações de uso e preservam-se os recursos naturais existentes, que incluem as áreas de preservação permanente. Odum (1988) define agroecossistema como "ecossistema agrícola que possui um componente autotrófico como parte integral, e difere dos ecossistemas naturais ou seminaturais, porque a diversidade de organismos é muito reduzida, em razão da ação antrópica para maximizar a produção de determinado alimento ou produto". O homem administra o solo, a vegetação e a água, e consome os produtos produzidos ou administrados. Os únicos seres produtores ou repositores no meio são os organismos fotossintetizantes. A atividade humana produz conseqüências positivas ou negativas no meio ambiente, e as geotecnologias aparecem como ferramentas que auxiliam no monitoramento, fiscalização, pesquisa e gestão dos recursos naturais.

O objetivo deste trabalho foi obter um mapa de favorabilidade à cafeicultura no ecossistema agrícola em estudo, e verificar se existe diferença quanto à favorabilidade entre quatro municípios do Estado de Minas Gerais.

\section{Material e Métodos}

A área de estudo localiza-se ao Sudoeste de Minas Gerais e compreende quatro municípios: Aguanil, Boa Esperança, Campo Belo e Cristais, o que corresponde à área total de $2.253 \mathrm{~km}^{2}$. Os municípios foram selecionados em razão da heterogeneidade da cultura do café, perante as características fisiográficas e o nível tecnológico de instalação das lavouras.

Foram utilizados neste estudo: o limite municipal na escala 1:100.000 (Companhia de Tecnologia da Informação do Estado de Minas Gerais, 1998); cartas topográficas na escala 1:50.000 (IBGE, 2005); mapa de solos na escala 1:100.000 (Barros, 2006); mapa de cafeicultura dos municípios (Barros, 2006). As variáveis utilizadas na modelagem AHP (drenagem, áreas de preservação permanente) foram extraídas das imagens dos sensores TM e CCD, a bordo dos satélites Landsat e Cbers, respectivamente (Instituto Nacional de Pesquisas Espaciais, 2005). As variáveis altimetria, declividade e orientação de vertentes foram obtidas da grade altimétrica do programa SRTM da National Aeronautics and Space Administration (2005), no trabalho de Barros (2006). Para a estruturação do banco de dados e tratamento das imagens, utilizaram-se os aplicativos Spring (Câmara et al., 1996) e Research Systems INC. (2004). Para o cálculo dos pesos hierárquicos das variáveis e a análise estatística, utilizaram-se os aplicativos Criterium Decision Plus (InfoHarvest, 2001) e Minitab (Minitab, 2000).

Por meio da integração das variáveis, o espaço geográfico foi parametrizado com a obtenção de um mapa geoambiental, segundo critério hierárquico para a tomada de decisão. A Figura 1 ilustra as etapas necessárias à obtenção do mapa geoambiental de favorabilidade à cultura do café.

Para aplicar o modelo AHP, foi estabelecida uma estrutura hierárquica entre as variáveis, em que o valor de dominância para um dado nível hierárquico é propagado para o nível inferior. A Figura 2 ilustra o processo de formulação da estrutura hierárquica, em função do problema e das variáveis utilizadas no processo de modelagem, estruturados em ambiente do aplicativo Criterium Decision Plus (InfoHarvest, 2001).

Para solucionar o problema apresentado na Figura 2, parte-se da hipótese de que o modelo AHP permite, a partir de variáveis fisiográficas e ambientais, construir um cenário de favorabilidade à cafeicultura nos quatro municípios estudados. Os critérios são as variáveis 
utilizadas no modelo, ponderadas de acordo com suas características agronômicas em relação às melhores condições à cafeicultura.

Para cada um dos critérios utilizados, estudou-se cada alternativa, tendo-se designado valores e pesos às classes de cada variável. Estruturados os critérios e as

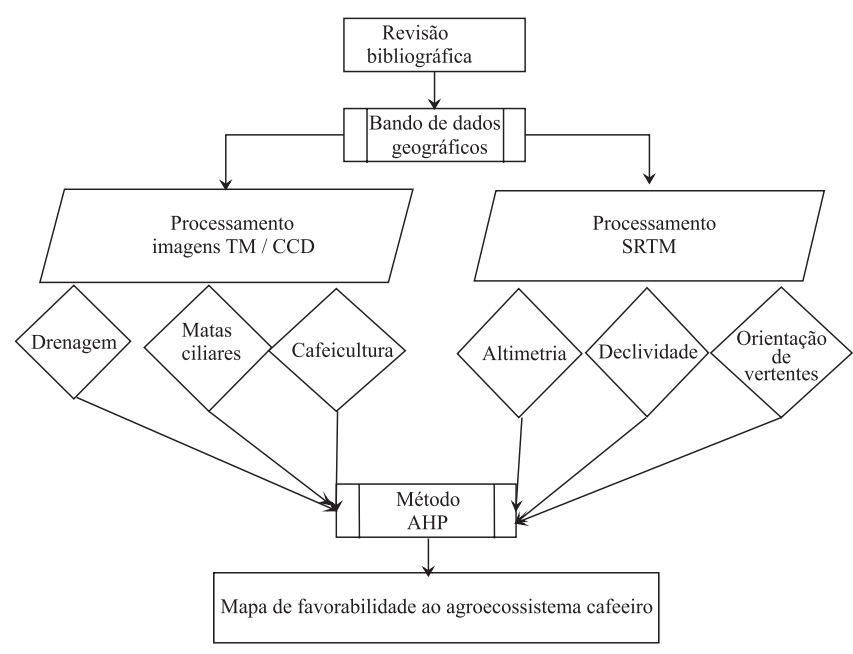

Figura 1. Fluxograma das etapas de desenvolvimento do trabalho. alternativas, foram realizadas comparações pareadas, para identificar quão melhor seria cada alternativa em relação às demais. Com base nesses critérios, foi possível realizar os julgamentos e definir os pesos utilizados na ponderação de cada variável. Nesse cenário, o valor zero corresponde à desfavorabilidade ao agroecossistema cafeeiro, e o valor um, à favorabilidade ao agroecossistema cafeeiro, a partir da hipótese formulada.

Para definir a hierarquização das variáveis, optou-se primeiro pela drenagem e matas ciliares, em razão da restrição legal à exploração dessas áreas. A segunda variável foi representada pelo mapa de solos na escala $1: 250.000$. Os parâmetros de fertilidade foram qualificados segundo análises químicas realizadas conforme Ministério da Agricultura (1962).

A terceira variável na hierarquia de ponderação foi a declividade, associada diretamente ao tipo de manejo da cultura, relacionada principalmente à mecanização das lavouras cafeeiras e conservação de solo. As variáveis altimetria e orientação de vertentes, indiretamente ligadas às condições climáticas, foram definidas como a quarta e quinta variável, respectivamente.

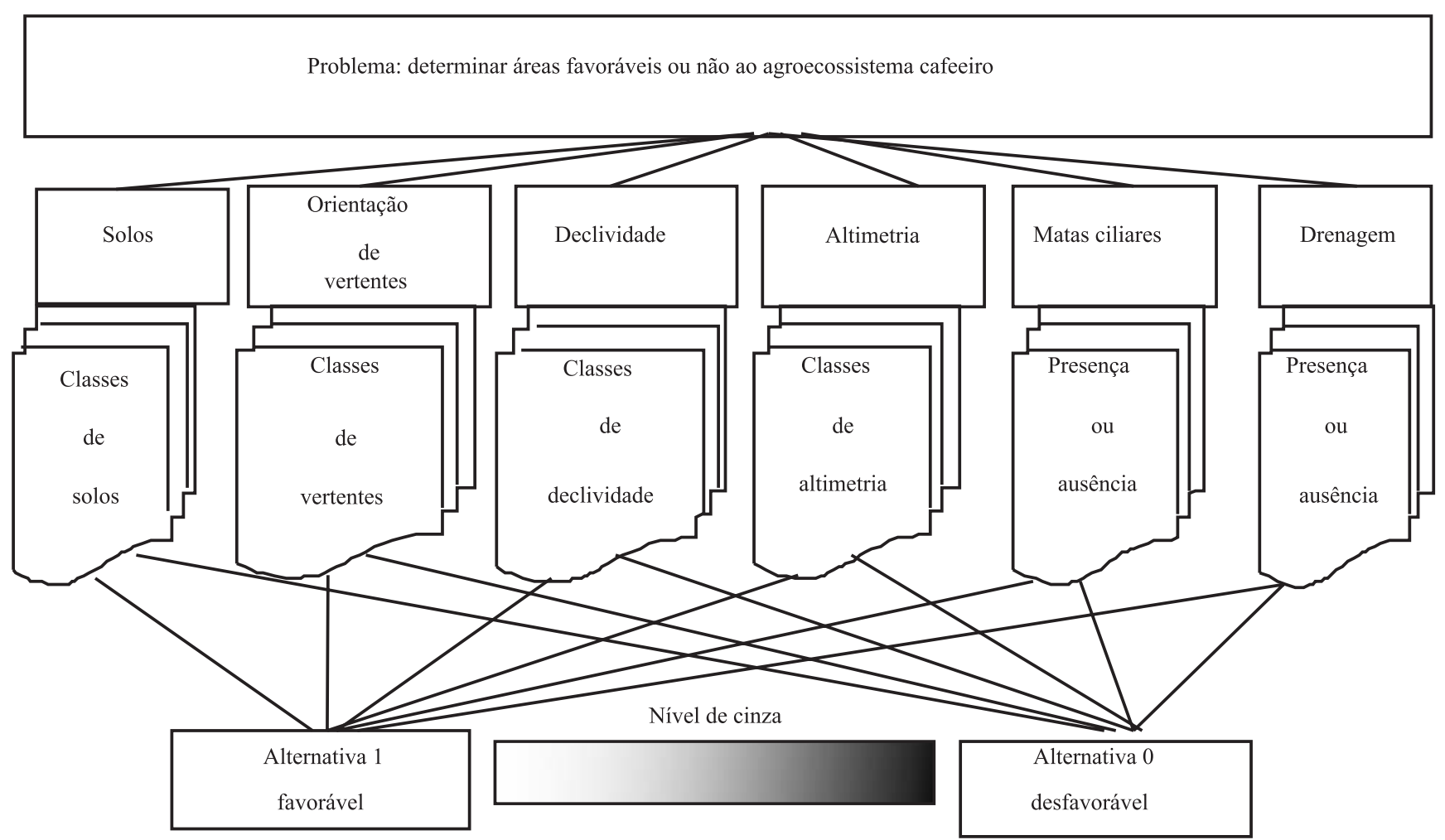

Figura 2. Estrutura hierárquica para determinar as áreas favoráveis ao agroecossistema cafeeiro. 
As premissas de favorabilidade ao cultivo do café, para cada uma das variáveis, foram obtidas em literatura e são descritas a seguir.

Drenagem + matas ciliares: proibida a exploração agropecuária sem outorga do órgão estadual ou federal competente (Brasil, 1965). Onde há drenagem ou área sob preservação permanente, considerou-se o espaço como desfavorável à cafeicultura, com peso das classes igual a zero. A manutenção dessas áreas intactas prioriza a preservação do ecossistema agrícola local e, por esse motivo, foi considerada como a mais importante no contexto da modelagem, com peso obtido igual a 0,638.

Solos: segundo Amaral et al. (1999), solos com limitações físicas (profundidade efetiva e estrutura) não são recomendados à exploração agrícola. Portanto, foram considerados inaptos à cafeicultura os solos identificados como Cambissolos, Neossolos e Gleissolos. Os solos intermediários com limitações físicas e químicas foram os Argissolos; finalmente, os solos de maior favorabilidade ao agroecossistema cafeeiro foram os Latossolos, e o peso atribuído foi 0,189. A distribuição dos pesos das classes ocorreu de acordo com atributos de fertilidade aparente ( $\mathrm{pH}, \mathrm{CTC}, \mathrm{V} \%)$, textura (arenosa, mista, argilosa) e profundidade efetiva (raso, médio e profundo), comparativamente às condições ideais obtidas em literatura. A partir de uma função linear, em que os atributos possuem a mesma importância relativa, obtevese um peso global para cada uma das classes de solo (Tabela 1).

Declividade: o critério da possibilidade de mecanização foi utilizado para a ponderação das classes de declividade.
Foram consideradas as seguintes classes de declividade, conforme Zambolim (2002): 0-20\%, favorável à mecanização, e acima de 20\%, impróprio à mecanização, tendo-se atribuído peso de 0,155 . A partir de uma função linear, em que os atributos de cada classe de solo possuem a mesma importância relativa, obteve-se peso global para cada uma das classes de declividade (Tabela 1).

Orientação de vertentes: no hemisfério sul, percebese que vertentes a nordeste recebem maior radiação solar durante a manhã, e vertentes a noroeste, maior radiação solar à tarde (Alzugaray \& Alzugaray, 1984). Recomenda-se o plantio de cafezais em vertentes orientadas a norte, nordeste e noroeste, com restrições ao plantio nas vertentes ao sul, sudeste e sudoeste, em razão da maior suscetibilidade a ventos frios e eventos climáticos adversos. Para esta classe, dividiu-se o intervalo entre 0 e 360 graus em oito classes, nas quais quatro classes são totalmente favoráveis à cafeicultura, e quatro classes desfavoráveis (Tabela 1). O peso calculado para a variável foi igual a 0,07 .

Altimetria: recomenda-se o plantio de cafezais em Minas Gerais, em faixa de altimetria entre $500 \mathrm{~m} \mathrm{e}$ $1.200 \mathrm{~m}$ (Sediyama et al., 2001). Com base nesta premissa, o peso calculado para essa variável foi igual a 0,034 . Os pesos individuais foram obtidos segundo função linear, de acordo com a proximidade dos valores das classes, em relação aos parâmetros ideais à instalação da cafeicultura.

$\mathrm{Na}$ estrutura de organização dos dados no modelo AHP, as variáveis são distribuídas em diferentes níveis

Tabela 1. Pesos globais das variáveis e pesos individuais em cada classe temática ponderada ${ }^{(1)}$.

\begin{tabular}{|c|c|c|c|c|}
\hline Drenagem & Altimetria (m) & Declividade (\%) & Vertentes & Solos \\
\hline Restritivo & $500<\mathrm{X}<1200$ & $(0-20)<Y>(21-100)$ & (NE-NW) & (Latossolos) \\
\hline Peso: 0,638 & Peso: 0,034 & Peso: 0,155 & Peso: 0,07 & Peso: 0,189 \\
\hline "app": 0 & $743-800 \mathrm{~m}=0,2$ & $0-10 \%=0,5$ & $\mathrm{E}-\mathrm{SEe}=0$ & Argissolo Amarelo: 0,1 \\
\hline \multirow[t]{8}{*}{ "rio": 0} & $801-850 \mathrm{~m}=0,2$ & $11-15 \%=0,25$ & $\mathrm{~N}-\mathrm{NE}=0,25$ & Argissolo Vermelho-Amarelo: 0,20 \\
\hline & $851-900 \mathrm{~m}=0,2$ & $16-20 \%=0,25$ & $\mathrm{NE}-\mathrm{E}=0,25$ & Argissolo Vermelho:0,20 \\
\hline & $901-950 \mathrm{~m}=0,2$ & $21-25 \%=0$ & $\mathrm{NW}-\mathrm{N}=0,25$ & Cambissolo Háplico: 0 \\
\hline & $951-1000 \mathrm{~m}=0,1$ & $26-50 \%=0$ & $\mathrm{~S}-\mathrm{SW}=0$ & Gleissolo + Organossolo: 0 \\
\hline & $1100-1200 \mathrm{~m}=0,1$ & $51-75 \%=0$ & $\mathrm{SE}-\mathrm{S}=0$ & Latossolo Vermelho-Amarelo: 0,25 \\
\hline & $1201-1300 \mathrm{~m}=0$ & $76-100 \%=0$ & $\mathrm{SW}-\mathrm{W}=0$ & Latossolo Vermelho:0,25 \\
\hline & $1301-1410 \mathrm{~m}=0$ & $>100 \%=0$ & $\mathrm{~W}-\mathrm{NW}=0,25$ & $\begin{array}{c}\text { Neossolo Litólico: } 0 \\
\text { Neossolo Quartzarênico: } 0\end{array}$ \\
\hline & $\Sigma$ pesos $=1$ & $\Sigma$ pesos $=1$ & $\Sigma$ pesos $=1$ & $\Sigma$ pesos $=1$ \\
\hline
\end{tabular}

${ }^{(1)}$ App: área de preservação permanente; E: leste, W: oeste, N: norte, S: sul. 
hierárquicos. Variáveis de maior importância transmitem sua maior influência para as variáveis menos importantes, em níveis hierárquicos mais baixos, que por sua vez contribuem também com a funcionalidade e coerência nos níveis superiores (Saaty, 1986). Para Alphonce (1997), o processo analítico hierárquico baseia-se em três princípios: decomposição, julgamentos comparativos e síntese de prioridades. Os valores atribuídos aos critérios e alternativas, na comparação pareada, são referentes a uma escala de medida de valores de intensidade ou de importância relativa. Esses valores, que variam de 0 a 1 , expressam a importância pela qual um critério ou alternativa é mais importante que o outro.

Com as variáveis organizadas e agrupadas, utilizouse o modelo proposto por Saaty (1986), para definir o grau de importância de cada uma das variáveis, em relação à restrição à exploração agrícola, utilizando-se uma lógica de comparação pareada. As variáveis foram comparadas duas a duas, e um grau de importância relativa foi atribuído ao relacionamento entre essas variáveis, conforme a escala fornecida por Alphonce (1997), em que: 1, as duas variáveis contribuem igualmente para o objetivo; 3 , uma variável é um pouco mais importante que a outra; 5, uma variável é claramente mais importante que a outra; 7 , uma variável é fortemente favorecida; 9 , a diferença entre as variáveis é da maior ordem possível; e 2, 4, 6 e 8 são valores intermediários entre os julgamentos das variáveis.

Selecionados os critérios para comparação, como também estabelecida a importância relativa de cada variável, o modelo AHP informa uma razão de consistência, que deve ser menor do que 0,1 (Alphonce, 1997). Esta razão de consistência atesta a coerência nos julgamentos realizados entre as variáveis.

Para a obtenção do modelo hierárquico, constroemse matrizes quadradas recíprocas positivas, cuja ordem será igual ao número de alternativas. Posteriormente, para cada critério ou subcritério faz-se o mesmo, progressivamente. Essas comparações por pares forneceram pesos para cada alternativa, dentro de cada critério ou subcritério, após comparações recíprocas. Esses pesos são obtidos por meio do cálculo do autovetor principal de cada matriz quadrada. Ao final do processo, esses pesos geraram uma função de agregação aditiva, na qual, para cada alternativa específica, atribuiu-se um valor final que possibilitou a ordenação global de todas as alternativas.

Os critérios utilizados foram obtidos com base em literatura e observações de campo. Nesse contexto, os maiores pesos das classes correspondentes a cada variável influíram com maior representatividade no mapa final. Os pesos utilizados no modelo foram obtidos via comparação pareada de variáveis, a partir do grau de importância (Tabela 1). Para o cálculo dos pesos, utilizouse o aplicativo Criterium Decision Plus (InfoHarvest, 2001).

Com as variáveis organizadas e agrupadas, o modelo AHP foi alimentado para o cálculo dos pesos de cada uma das variáveis e, finalmente, o resultado foi espacializado via aplicativo Spring (Câmara et al., 1996). Como produto final, obteve-se uma matriz numérica com intervalo entre 0 e 1 . A equação abaixo se refere à função matemática utilizada para representação espacial do modelo, no qual $\mathrm{X}$ representa uma grade ou matriz numérica com os valores do modelo calculados entre 0 e 1 .

$\mathrm{X}=0,034($ altimetria $)+0,155($ declividade $)+$ $0,638$ (drenagem + app $)+0,189$ (solos) $+0,070$ (vertentes)

Para a análise dos resultados, aplicou-se o teste Shapiro-Wilk, para verificar a normalidade dos dados calculados no mapa de favorabilidade à cafeicultura, em escala municipal. Utilizou-se uma amostragem aleatória dos valores em nível municipal, proporcionais ao tamanho de cada um dos municípios. Refutada a hipótese de normalidade, bem como a necessidade de uma análise da variância dos dados, aplicou-se o teste de Kolmogorov-Smirnov. Este teste, não paramétrico, foi realizado para comparar amostras finitas obtidas aleatoriamente, em cada um dos municípios, e confirmar ou refutar a hipótese de que as amostras coletadas para todos os municípios possuem a mesma função de distribuição de probabilidade, considerando-se dois níveis de significância (1 e 5\%), o que permite verificar possível diferença entre os municípios, em relação à favorabilidade à cafeicultura.

Considerando-se que o número de amostras para esta análise deve ser o mesmo, conforme estabelecido no teste de Kolmogorov-Smirnov, foi selecionado o Município de Aguanil, em razão do menor universo amostral (358 indivíduos). Além disso, como a análise deste estudo estava restrita a apenas quatro municípios e não havia restrição ao processamento do número de amostras, selecionou-se o maior número de amostras possível (99\%), no total de 356 amostras para cada município.

Para a análise estatística da favorabilidade ao agroecossistema, nas áreas cultivadas com café no ano de 2004, data base do mapa utilizado, foram selecionadas 121 amostras (98\%), com base no Município de Aguanil, que possui o menor universo amostral (123 indivíduos). As amostras são compostas pela menor unidade que compõe o mapa (pixel). 


\section{Resultados e Discussão}

O método utilizado permitiu associar, em um único mapa, todas as variáveis utilizadas na determinação de áreas favoráveis à cafeicultura. Áreas com solos limitantes, associados à alta declividade e altimetria acima de $1.200 \mathrm{~m}$, indicam o predomínio do processo de morfogênese que induz naturalmente a processos erosivos. Nestes casos, observou-se no mapa geoambiental que os níveis de cinza representados são menores, conforme escala utilizada, principalmente na
Serra da Boa Esperança, Município de Boa Esperança (Figura 3). As áreas com maior favorabilidade, ou seja, com maior nível de cinza, podem ser observadas no centro-sul do Município de Boa Esperança e noroeste do Município de Cristais.

A coerência do mapa geoambiental obtido está visivelmente expressada pelos menores valores de nível de cinza, em áreas da represa de Furnas, rios, córregos e solos com limitações físicas (Figura 3). As áreas de drenagem e preservação permanente são desfavoráveis ao agroecossistema cafeeiro. Outras áreas, com nível

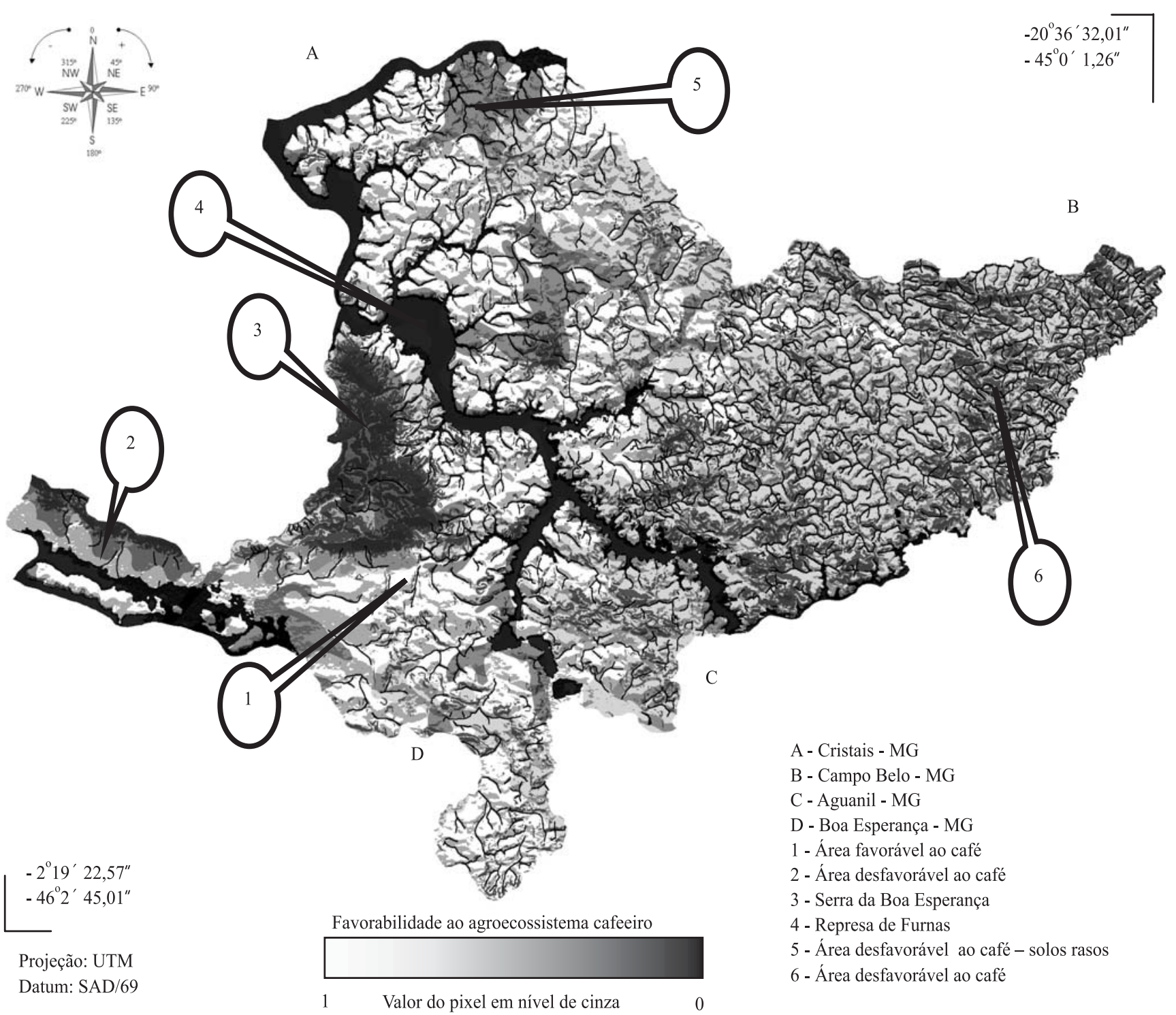

Figura 3. Mapa de favorabilidade ao agroecossistema cafeeiro. 
de cinza próximo a 0 , estão relacionadas à presença de solos rasos e declividade acentuada (Figura 3). As áreas favoráveis à cafeicultura possuem valores de cinza próximos a 1 .

Com base no resultado do teste de normalidade (Shapiro-Wilk), constatou-se que em todos os municípios refutou-se a hipótese de normalidade para os valores de favorabilidade da grade numérica (Tabela 2). Observouse que todos os valores de $\mathrm{W}$ calculados foram menores que os valores de $\mathrm{W}$ críticos, e o valor de probabilidade foi igual a zero, com 1 e $5 \%$ de significância, respectivamente.

Para a análise da favorabilidade à cafeicultura em escala municipal, observou-se com 5\% de significância que todos os municípios diferem entre si (Tabela 3), o que atesta a variabilidade fisiográfica local relatada no trabalho de Barros (2006).

Tabela 2. Resultado do teste de normalidade de Shapiro-Wilk, com os valores $\mathrm{W}$ calculados (W calc) e W críticos (W x) e o respectivo valor de probabilidade $(\mathrm{p})$.

\begin{tabular}{lclc}
\hline População & W calc & W x & p \\
\hline Aguanil (2.000 amostras) & 0,656 & $0,90^{*}$ & 0,000 \\
& & $0,92 * *$ & \\
Boa Esperança (5.000 amostras) & 0,515 & $0,90^{*}$ & 0,000 \\
Campo Belo (4.000 amostras) & 0,3621 & $0,92 * *$ & \\
& & $0,90^{*}$ & 0,000 \\
Cristais (4.500 amostras) & 0,564 & $0,90 *$ & \\
& & $0,92 * *$ & 0,000 \\
\hline
\end{tabular}

* e**Significativo a 5 e $1 \%$ de probabilidade, respectivamente.
Para as áreas cultivadas com café, o teste de Kolmogorov-Smirnov indicou, com 5\% de significância, que as condições de favorabilidade entre Boa Esperança e Cristais são iguais (valor de probabilidade $=22,37 \%$ ) (Tabela 4). Aguanil possui as mesmas condições de favorabilidade que o Município de Boa Esperança, considerando-se um valor de probabilidade de $4 \%$. Campo Belo possui as mesmas condições de favorabilidade que Aguanil (valor de probabilidade $=1,19 \%$ ). A análise estatística permitiu concluir que as áreas cafeeiras de Boa Esperança e Cristais são aquelas que mais se assemelham em condições de cultivo, o que corrobora as observações de Barros (2006).

A maior dificuldade em relação à aplicação do modelo AHP está relacionada à determinação dos pesos das variáveis e das suas classes, problema comumente encontrado em outros trabalhos que utilizaram este método (Thirumalaivasan et al., 2003; Li et al., 2005). Silva \& Belderrain (2005) destacaram como principal limitação do modelo AHP o problema de inversão de ordem das alternativas. Isto ocorre se uma alternativa for adicionada ou removida da análise, o que provoca uma inversão nos resultados. Apesar da independência requerida entre os níveis hierárquicos, há dependência funcional que o especialista infringirá, caso não a considere na formulação da hipótese e solução do problema. Os mesmos autores sugerem, para remediar o processo de inversão, a utilização de uma escala absoluta, pois a composição final dos pesos permanecerá eqüitativa e não afetará outras análises.

Tabela 3. Teste de Kolmogorov-Smirnov, na comparação das médias de favorabilidade ao agroecossistema cafeeiro a 5\% de probabilidade $^{(1)}$.

\begin{tabular}{|c|c|c|c|c|c|c|}
\hline \multirow[t]{2}{*}{ Município } & \multicolumn{2}{|c|}{ Cristais } & \multicolumn{2}{|c|}{ Campo Belo } & \multicolumn{2}{|c|}{ Aguanil } \\
\hline & Hipótese & $\begin{array}{c}\text { Valor de } \\
\text { probabilidade }\end{array}$ & Hipótese & $\begin{array}{c}\text { Valor de } \\
\text { probabilidade }\end{array}$ & Hipótese & $\begin{array}{c}\text { Valor de } \\
\text { probabilidade }\end{array}$ \\
\hline Boa Esperança & 1 & 0,0000 & 1 & 0,0000 & 1 & 0,0000 \\
\hline Cristais & - & - & 1 & 0,0000 & 1 & 0,0000 \\
\hline Campo Belo & 1 & 0,0000 & - & - & 1 & 0,0000 \\
\hline
\end{tabular}

${ }^{(1)}$ Hipótese: $\mathrm{Ho}=0$ (valores médios de favorabilidade iguais); $\mathrm{Ha}=1$ (valores médios de favorabilidade diferentes).

Tabela 4. Teste de Kolmogorov-Smirnov, na comparação das médias de favorabilidade ao agroecossistema cafeeiro, nas lavouras de café em produção, a $5 \%$ de probabilidade $^{(1)}$.

\begin{tabular}{|c|c|c|c|c|c|c|}
\hline \multirow[t]{2}{*}{ Município } & \multicolumn{2}{|c|}{ Cristais } & \multicolumn{2}{|c|}{ Campo Belo } & \multicolumn{2}{|c|}{ Aguanil } \\
\hline & Hipótese & $\begin{array}{c}\text { Valor de } \\
\text { probabilidade }\end{array}$ & Hipótese & $\begin{array}{c}\text { Valor de } \\
\text { probabilidade }\end{array}$ & Hipótese & $\begin{array}{c}\text { Valor de } \\
\text { probabilidade }\end{array}$ \\
\hline Boa Esperança & 0 & 0,2237 & 1 & 0,0000 & 1 & 0,0434 \\
\hline Cristais & - & - & 1 & 0,0000 & 1 & 0,0056 \\
\hline Campo Belo & 1 & 0,0000 & - & & 1 & 0,0119 \\
\hline
\end{tabular}

(1)Hipótese: Ho = 0 (valores médios de favorabilidade iguais); Ha = 1 (valores médios de favorabilidade diferentes). 
Constatou-se que o método utilizado é muito dependente do analista e do problema, e tem sido alvo de críticas, pois permite gerar uma escala de razão de preferências por parte do especialista.

\section{Conclusões}

1. O processo analítico hierárquico (AHP) permite determinar as áreas potencialmente favoráveis à cafeicultura no agroecossistema em estudo.

2. Os municípios diferem entre si quanto à favorabilidade da cafeicultura no agroecossistema.

\section{Referências}

ALPHONCE, C.B. Application of the analytic hierarchy process in agriculture in developing countries. Agricultural Systems, v.53, p.97-112, 1997.

ALZUGARAY, D.; ALZUGARAY, C. Flora brasileira. São Paulo: Três Livros, 1984.

AMARAL, F.C.S.; PEREIRA, N.R.; CARVALHO JUNIOR, W. Principais limitações dos solos brasileiros. Rio de Janeiro: Embrapa Solos, 1999. Disponível em: www.cnps.embrapa.br/serch/ pesqs/tema3/tema3.html. Acesso em: 15 jul. 2005.

BARROS, M.A. Geotecnologias como contribuição ao estudo do Agroecossistema cafeeiro de Minas Gerais em nível municipal. 2006. 157p. Dissertação (Mestrado) - Instituto Nacional de Pesquisas Espaciais, São José dos Campos.

BRASIL. Decreto-lei no 4771, de 15 de setembro de 1965. Institui o novo Código Florestal. Diário Oficial [da] República Federativa do Brasil. Brasília, 16 set. 1965. Disponível em: https:// legislacao.planalto.gov.br/legisla/legislacao.nsf/ fraWeb?OpenFrameSet $\&$ Frame $=$ frmWeb2 $\&$ Src $=/$ legisla $/$ legislacao.nsf\%2FFrmConsultaWeb1\%3FOpenForm\%26AutoFramed. Acesso em: 23 nov. 2007.

BRASIL. Ministério da Agricultura. Levantamento de reconhecimento dos solos da região sob influência do reservatório de Furnas. Rio de Janeiro: CNEPA-SNPA, 1962. 462p. (SNPA - Boletim Técnico, 37).

CÂMARA, G.; MEDEIROS, J.S. Princípios básicos em geoprocessamento. In: ASSAD, E.D.; SANO, E.E. Sistema de informações geográficas: aplicações na agricultura. Planaltina: Embrapa-CPAC, 1993. p.3-11.

CÂMARA, G.; MONTEIRO, A.M.V.; MEdeiRos, S.A. Introdução à ciência da geoinformação: fundamentos epistemológicos da ciência da geoinformação. Disponível em: http:/ /www.dpi.inpe.br/gilberto/livro/introd/cap5-epistemologia.pdf. Acesso em: 16 jan. 2007.

CÂMARA, G.; SOUZA, R.C.M.; FREITAS, U.M.; GARRIDO, J. Spring: integrating remote sensing and GIS by object-oriented data modelling. Computers \& Graphics, v.20, p.395-403, 1996.
COMPANHIA DE TECNOLOGIA DA INFORMAÇÃO DO ESTADO DE MINAS GERAIS. Limite dos Municípios de Minas Gerais. Belo Horizonte, 1998. Disponível em: http:// www.geominas.mg.gov.br/. Acesso em: 15 mar. 2005.

CREPANI, E.; MEDEIROS, J.S. Imagens fotográficas derivadas de MNT do projeto SRTM para fotointerpretação na geologia, geomorfologia e pedologia. São José dos Campos: Inpe, 2004. 39p.

CREPANI, E.; MEDEIROS, J.S.; HERNANDEZ FILHO, P.H.; FLORENZANO, T.G.; DUARTE, V.; BARBOSA, C.C.F. Sensoriamento remoto e geoprocessamento aplicados ao zoneamento ecológico-econômico e ao ordenamento territorial. São José dos Campos: Inpe, 2001. 101p.

FELGUEIRAS, C.A.; ERTHAL, G.J. Aplicações de modelos numéricos de elevação e integração com imagens digitais. In: SIMPÓSIO BRASILEIRO DE SENSORIAMENTO REMOTO, 5., Natal. Anais. São José dos Campos: Inpe, 1988. p.485-490.

GUO, L.S.; HE, Y.S. Integrated multi-criterial decision model: a case study for the allocation of facilities in Chinese agriculture. Journal of Agricultural Engineering Research, v.73, p.87-94, 1999.

INFOHARVEST. Criterium decision plus 3.04. Seattle: InfoHarvest, 2001.

INSTITUTO BRASILEIRO DE GEOGRAFIA E ESTATÍSTICA. Folha Campo Belo. SF-23-Y-D-II-1 MI-2769/1. Brasília: IBGE, 2005. 3 Mapas. Escala 1:50.000. Disponível em: http://www.ibge.gov.br. Acesso em: 12 mar. 2005.

INSTITUTO NACIONAL DE PESQUISAS ESPACIAIS. Catálogo de imagens. São José dos Campos: Inpe, 2005. Disponível em: http://www.dgi.inpe.br/CDSR. Acesso em: 15 abr. 2005.

KARAMI, E. Appropriateness of farmers' adoption of irrigation methods: the application of the AHP model. Agricultural Systems, v.87, p.101-119, 2006.

KURKDJIAN, M.L.N.O.; VALÉRIO FILHO, M.; VENEZIANI, P.; PEREIRA, M.N.; FLORENZANO, T.G.; ANJOS, C.E.; OHARA, T.; DONIZETI, P.L.; ABDON, M.M. Macrozoneamento da região do Vale do Paraíba e Litoral Norte do Estado de São Paulo. São José dos Campos, Inpe, 1992. 176p.

LI, X.; MIN, M.; TAN, C. The functional assessment of agricultural ecosystems in Hubei Province, China. Ecological Modelling, v.187, p.352-360, 2005.

MATIELLO, J.B. Café: do cultivo ao consumo. São Paulo: Globo, 1991. 320p.

MINITAB. Minitab Statistical Tab. Pennsylvânia: [s.n.], 2000. 1 CD-ROM.

NATIONAL AERONAUTICS AND SPACE ADMINISTRATION. Shuttle Radar Topography Mission (SRTM) data product: SRTMS21W046 \& S22W046. California, 2005. Disponível em: http:// www2.jpl.nasa.gov/srtm/dataprelimdescriptions.html. Acesso em: 20 jan. 2005.

ODUM, E.P. Ecologia. Rio de Janeiro: Guanabara Koogan, 1988. $446 \mathrm{p}$. 
RESEARCH SYSTEMS INC. The Environment for Visualizing Images (ENVI) 4.1. Boulder: Research Systems, 2004.

SAATY, T.L. Axiomatic foundation of the analytic hierarchy process. Management Science, v.32, p.841-855, 1986.

SEDIYAMA, G.C.; MELO JUNIOR, J.C.; SANTOS, A.R.; RIBEIRO, A.; COSTA, M.H.; HAMAKAWA, P.J.; COSTA, J.M.N.; COSTA, L.C. Zoneamento agroclimático do cafeeiro (Coffea arabica L.) para o Estado de Minas Gerais. Revista Brasileira de Agrometeorologia, v.9, p.501-509, 2001.

SILVA, R.M.; BELDERRAIN, M.C.N. Considerações sobre métodos de decisão multicritério. São José dos Campos: ITA, 2005. Disponível em: http://www.bibl.ita.br/xiencita/Artigos/ Mec03.pdf . Acesso em: 21 nov. 2007.
THIRUMALAIVASAN, D.; KARMEGAM, M.; VENUGOPAL, K. AHP-Drastic: software for specific aquifer vulnerability assessment using drastic model and GIS. Environmental Modelling \& Software, v.18, p.645-656, 2003.

VENTURIERI, A. Utilização da segmentação de imagens e lógica nebulosa para treinamento de uma rede neural artificial na caracterização de classes de uso da terra na região de TucuruíPA. 1995. 111p. Dissertação (Mestrado) - Instituto Nacional de Pesquisas Espaciais, São José dos Campos.

ZAMBOLIM, L. (Ed.). Tecnologia de produção de café com qualidade. Viçosa: Universidade Federal de Viçosa, 2002. 648p.

ZHANG, B.; ZHANG, Y.; CHEN, D.; WHITE, R.E.; LI, Y. A quantitative evaluation system of soil productivity for intensive agriculture in China. Geoderma, v.123, p.319-331, 2004.

Recebido em 11 de junho de 2007 e aprovado em 21 de novembro de 2007 